\title{
BMJ Open Perioperative urinary thromboxane metabolites and outcome of coronary artery bypass grafting: a nested case- control study
}

\author{
Hanning Liu, ${ }^{1}$ Zhengxi Xu, ${ }^{1}$ Cheng Sun, ${ }^{1}$ Qianlong Chen, ${ }^{1,2}$ Ning Bao, ${ }^{1}$ Wen Chen, ${ }^{1,2}$
} Zhou Zhou, ${ }^{1,2}$ Xiaoqi Wang, ${ }^{1}$ Zhe Zheng ${ }^{1}$

To cite: Liu H, Xu Z, Sun C, et al. Perioperative urinary thromboxane metabolites and outcome of coronary artery bypass grafting: a nested case-control study. BMJ Open 2018;8:e21219. doi:10.1136/ bmjopen-2017-021219

- Prepublication history and additional material for this paper are available online. To view these files, please visit the journal online (http://dx.doi. org/10.1136/bmjopen-2017021219).

$H L$ and $Z X$ contributed equally. $\mathrm{ZZ}, \mathrm{XW}$ and $\mathrm{ZZ}$ contributed equally.

Received 22 December 2017 Revised 8 June 2018 Accepted 19 July 2018

A Check for updates

C C Author(s) (or their employer(s)) 2018. Re-use permitted under CC BY-NC. No commercial re-use. See rights and permissions. Published by BMJ.

${ }^{1}$ State Key Laboratory of Cardiovascular Disease, Fuwai Hospital, National Center for Cardiovascular Diseases, Chinese Academy of Medical Sciences and Peking Union Medical College, Beijing, China ${ }^{2}$ Beijing Key Laboratory for Molecular Diagnostics of Cardiovascular Diseases, Fuwai Hospital, Beijing, China

Correspondence to

Dr Zhe Zheng;

zhengzhe@fuwai.com

\section{ABSTRACT}

Objective As a marker of in vivo thromboxane generation, high-level urinary thromboxane metabolites (TXA-M) increase the occurrence of cardiovascular events in highrisk patients. To investigate whether perioperative urinary TXA-M level is associated with major adverse cardiac and cerebrovascular events (MACCE) after coronary artery bypass graft (CABG) surgery, we designed a nested casecontrol study.

Design Observational, nested case-control study. Setting Single-centre outcomes research in Fuwai Hospital, Beijing, China.

Participants One thousand six hundred and seventy Chinese patients undergoing CABG surgery from September 2011 to October 2013.

Methods We obtained urinary samples from 1670 Chinese patients undergoing CABG 1 hour before surgery (pre-CABG), and 6 hours (post-CABG 6 hours) and 24 hours after surgery (post-CABG 24 hours). Patients were followed up for 1 year, and we observed 56 patients had MACCE. For each patient with MACCE, we matched three control subjects. Perioperative urinary TXA-M of the three time spots was detected in these 224 patients.

Results Post-CABG 24 hours TXA-M is significantly higher than that of patients without MACCE (11101vs8849 pg/mg creatine, $\mathrm{P}=0.007$ ). In addition, patients in the intermediate tertile and upper tertile of post-CABG 24 hours urinary TXA-M have a 2.2 times higher ( $\mathrm{HR} 2.22,95 \% \mathrm{Cl} 1.04$ to $4.71, \mathrm{P}=0.038)$ and a 2.8 times higher (HR $2.81,95 \% \mathrm{Cl}$ 1.35 to $5.85, P=0.006$ ) risk of 1 year MACCE than those in the lower tertile, respectively.

Conclusions In conclusion, post-CABG 24 hours urinary TXA-M elevation is associated with an increase of 1 year adverse events after CABG, indicating that the induction of cyclo-oxygenase- 2 by surgery-related inflammatory stimuli or platelet turnover may be responsible for the high levels of post-CABG urinary TXA-M.

Trial registration number NCT01573143.

\section{INTRODUCTION}

Coronary artery disease (CAD) is the leading cause of mortality worldwide. As one of the commonly used revascularisation strategies, coronary artery bypass graft (CABG) surgery
Strengths and limitations of this study

- We used a nested case-control design to analyse the association between perioperative urinary thromboxane metabolite (TXA-M) and major adverse cardiac and cerebrovascular events (MACCE) in a coronary artery bypass graft (CABG) cohort. This $\mathrm{CABG}$ cohort was from a randomised controlled trial (STICS), so the cases (56 patients with MACCE) and the 168 controls from the same cohort had the comparable baseline characteristics, which made our results more reliable and powerful.

- We tested the TXA-M levels at different time points before and after surgery establishing more reliable association between the TXA-M levels and adverse events after surgery.

- We have not tested TXA-M levels at later time points beyond the first day after CABG, thus, whether the TXA-M level in this study is a temporary or stable change should be verified in further studies.

is the standard of care for patients with CAD with diabetes or multivessel CAD. ${ }^{1}$ In the first year after CABG, thrombotic dysfunction, for example, thrombus occlusion of saphenous vein grafts $(\mathrm{SVG}),{ }^{2}$ is one of the main reasons increasing the risk of adverse events, including death, myocardial infarction (MI) and repeat revascularisation. ${ }^{34}$

Thromboxane $\mathrm{A}_{2}\left(\mathrm{TXA}_{2}\right)$ is an unstable metabolite of arachidonic acid (AA). Numerous studies have revealed $\mathrm{TXA}_{2}$ as a culprit of cardiovascular diseases. ${ }^{5-\tau^{2}}$ As a platelet agonist, $\mathrm{TXA}_{2}$ activates adjacent platelets, provokes more platelet-dependent $\mathrm{TXA}_{2}$ generation, and thus triggers platelet aggregation. ${ }^{8}$ Under normal conditions, $\mathrm{TXA}_{2}$ is dominantly synthesised by platelet via cyclo-oxygenase-1 pathway in humans. Aspirin, inhibiting platelet cyclo-oxygenase-1, has been commonly used as an antiplatelet therapy to reduce $\mathrm{TXA}_{2}$ generation and prevent secondary vascular thrombotic 
events. While, under the condition of acute inflammatory stimuli, such as surgery or cardiopulmonary bypass, the expression level of cyclo-oxygenase-2 can increase dramatically and promote the synthesis of $\mathrm{TXA}_{2}$, the CABG procedure enhances inflammatory process and platelet turnover, which causes the elevation of $\mathrm{TXA}_{2}$ by the induction of cyclo-oxygenase-2. ${ }^{9}$ Previous studies have reported that $\mathrm{TXA}_{2}$ generation was an independent risk factor for early SVG thrombosis after CABG. ${ }^{4}$

However, whether perioperative $\mathrm{TXA}_{2}$ generation is associated with adverse events after CABG is unclear. We hypothesise that enhanced perioperative $\mathrm{TXA}_{2}$ generation is correlated with poor prognosis after CABG. We studied a cohort of 1670 patients with CABG from the Statin Therapy in Cardiac Surgery (STICS) trial (ClinicalTrials.gov number, NCT01573143) ${ }^{10}$ with a nested case-control analysis, to determine whether perioperative $\mathrm{TXA}_{2}$ generation, measured by urinary thromboxane metabolites (TXA-M), is correlative with major adverse cardiac and cerebrovascular events (MACCE), including all-cause death, non-fatal MI, non-fatal stroke and repeat revascularisation after $\mathrm{CABG}$.

\section{METHODS}

\section{Study design}

We have complied with the World Medical Association Declaration of Helsinki regarding ethical conduct of research involving human subjects and/or animals. All patients provided written informed consent to be involved in the study.

\section{Patient enrolment}

Patients were selected from the STICS trial. ${ }^{10}$ Between September 2011 and October 2013, 1922 patients at Fuwai hospital were randomised, among which 1670 underwent CABG (supplementary methods. For all patients, the medication of ASA stopped at least 5 days before CABG and postoperatively, starting within the first 24 hours postoperation $(100 \mathrm{mg})$. All patients were required to come back for a routine outpatient follow-up visit at 1 month, 6 months and each year after discharge. The medical records of those who reported any adverse events after discharge were reviewed for further confirmation. The primary end point of follow-up was a composite of MACCE (ie, non-fatal stroke, non-fatal MI, repeat revascularisation or death from any cause) ${ }^{11} 12$ (supplementary methods). Among the 1670 patients undergoing CABG, $15(0.9 \%)$ were lost during the first year follow-up, and 56 out of 1655 followed-up patients $(3.4 \%)$ had MACCE. With the use of a nested case-control analysis, we matched each patient with MACCE with three controls from the cohort; matching was based on sex, age, body mass index, hypertension, diabetes mellitus, smoking status, ejection fraction, COPD, previous MI, previous stroke, chronic kidney disease and peripheral vascular disease. Medication use after discharge was recorded at 1 year follow-up; for patients who missed this information we used prescription at discharge instead.

\section{Sample collection and preparation}

For every patient enrolled in this study, urinary samples were collected with cryogenic vials at three time spots: 1 hour before CABG (pre-CABG), 6 hours after CABG (post-CABG 6 hour) and twenty-four hours after CABG (post-CABG 24 hours). All urinary samples were stored at $-80^{\circ} \mathrm{C}$. Before testing, samples were thawed at $4^{\circ} \mathrm{C}$ for an hour and then centrifuged at $1000 \mathrm{~g}$ for $15 \mathrm{~min}$. Finally, $100 \mathrm{ul}$ from the supernatant was collected for further analysis.

\section{Patient and public involvement}

All data in this study were from the STICS trial (NCT01573143), so the present study did not involve participants and/or public in the study design and we obtained no more information or biological samples from patients in this study. Results will be disseminated to study participants via this publication.

\section{Measurement of TXA ${ }_{2}$ generation}

As $\mathrm{TXA}_{2}$ is an unstable metabolite, we measured urinary TXA-M, including 11-dehydro-thromboxane $\left(\mathrm{TXB}_{2}\right.$ and 11-dehydro-2, 3-dinor $\mathrm{TXB}_{2}$ using the AspirinWorks 11-dehydro-TXB ${ }_{2}$ ELISA (Corgenix, Broomfield, Colorado, USA) and expressed as a ratio to urinary creatine as previously described. ${ }^{13}$ The measurement was performed during December 2014-Janurary 2015.

\section{Statistical analysis}

For demographic description of patients, we calculated the means $( \pm \mathrm{SD})$ for continuous variables in both the MACCE and the control groups and compared them using Student's t-test; differences in discontinuous variables were evaluated using $\chi^{2}$ test. For TXA-M level, we used the median and IQR. Comparison of TXA-M levels between the two groups was conducted by Mann-Whitney analysis because its distribution was non-normally distributed. For survival analysis, pre-CABG, post-CABG 6 hours and post-CABG 24 hours urinary TXA-M were divided into tertiles according to their quantitative levels, respectively. The correlation between MACCE and TXA-M levels were estimated using the KaplanMeier method and log-rank test. Univariate and multivariate Cox proportional hazards regression models were used to estimate HRs and 95\% CIs. Moreover, we adjusted the model by multiple variables listed in online supplementary table S1, all of which were reported to correlate with the pathogenesis and prognosis of CAD significantly and C-statistic calculations were used for logistic regression. All statistical analyses were done with SPSS V.19.0 for Windows (SPSS, Chicago, Illinois, USA).

\section{RESULTS}

\section{Study population characteristics}

The STICS trial includes 1670 patients undergoing CABG. During the first year of follow-up after CABG, 15 of them were lost. Among the remaining 1655 patients (99.1\%), 


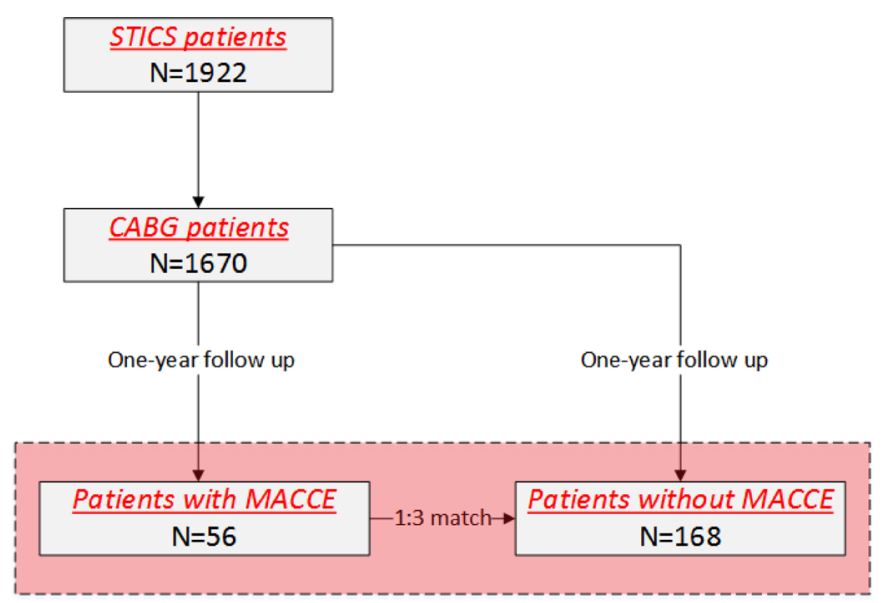

Figure 1 Flow chart of study design. We recruited 1670 patients with CABG from the STICS trial. After 1 year followup, 56 patients had MACCE. With the use of a nested casecontrol analysis, we matched each patient with MACCE with three controls from the cohort; thus, a cohort of 224 patients was established for further study. CABG, coronary artery bypass graft; MACCE, major adverse cardiac and cerebrovascular events; STICS, Statin Therapy in Cardiac Surgery.

56 had MACCE (3.4\%) (figure 1). For every patient with MACCE, we matched three controls without MACCE for further analysis. The baseline of the MACCE and the control groups has been presented in table 1 . The average age of patients with MACCE was 61.7 years, and 44 out of 56 patients with MACCE (78.6\%) were male. There were no differences in age, sex, body mass index, hypertension, diabetes mellitus, smoking status, COPD, peripheral vascular diseases, previous MI, chronic kidney diseases and ejection fraction between the MACCE and the control groups. In this nested case-control study, 26 $(46.4 \%)$ patients in the MACCE group and $84(50.0 \%)$ in the non-MACCE group were treated by perioperative rosuvastatin according to the study protocol of the STICS trial. After discharge, all the secondary preventive medications including aspirin, $\beta$-blockers, statins, ACE inhibitors and calcium channel blockers showed no differences between patients with MACCE and controls.

\section{Perioperative TXA-M generation between the MACCE and the control groups}

To determine whether the perioperative TXA-M generation was associated with 1 year MACCE after CABG, we tested pre-CABG, post-CABG 6hours and post-CABG 24hours urinary TXA-M levels. In both, the MACCE and the control groups, the generation of TXA-M was elevated after CABG. Urinary TXA-M generation before CABG was $5076 \mathrm{pg} / \mathrm{mg}$ creatine in the control group, which was slightly higher than that in the MACCE group $(4540 \mathrm{pg} /$ $\mathrm{mg}$ creatine, $\mathrm{P}=0.047)$. The levels of TXA-M post-CABG 6 hours in patients with MACCE and controls showed no significant difference (24016 vs $25681 \mathrm{pg} / \mathrm{mg}$ creatine, $\mathrm{P}=0.727$ ). However, TXA-M post-CABG 24 hours of patients with MACCE was significantly higher than that of patients without MACCE (11 101 vs $8849 \mathrm{pg} / \mathrm{mg}$ creatine, $\mathrm{P}=0.007$ ) (table 2).

Furthermore, we compared perioperative TXA-M generation in patients who died, patients with stroke, patients with $\mathrm{MI}$ and patients with repeat revascularisation with perioperative TXA-M generation in controls. Pre-CABG and post-CABG 6-hours urinary TXA-M generation showed no differences between patients who died, patients with stroke, patients with MI, patients with repeat revascularisation and controls. Post-CABG 24hours TXA-M after CABG was significantly higher in patients who died (11993 vs $8849 \mathrm{pg} /$ mg creatine, $\mathrm{P}=0.039$ ) and patients with stroke (11138 vs $8849 \mathrm{pg} / \mathrm{mg}$ creatine, $\mathrm{P}=0.016)$. In addition, no differences were detected between patients with repeat revascularisation and controls in post-CABG 24hours generation of TXA-M (table 3).

\section{Survival analysis of perioperative TXA-M levels and MACCE}

Next, we performed survival analysis of perioperative TXA-M levels and MACCE. We divided pre-CABG (tertile 1: 3750; tertile 2: 3750 6150; tertile 3: 6150 , pg/mg creatine), postCABG 6hours (tertile 1: 19750; tertile 2: 19750 30300; tertile 3: 30300 , pg/mg creatine) and post-CABG 24 hours (tertile 1: 7000; tertile 2: 7000 12000; tertile 3: 12000 , $\mathrm{pg} / \mathrm{mg}$ creatine) urinary TXA-M into tertiles according to their quantitative levels.

There were no significant differences of MACCE risk regarding to tertiles of pre-CABG $\left(\mathrm{P}_{\text {trend }}=0.075\right)$ and postCABG 6hours urinary TXA-M $\left(\mathrm{P}_{\text {trend }}=0.755\right)$. However, post-CABG 24hours TXA-M was significantly associated with 1 year MACCE $\left(\mathrm{P}_{\text {trend }}=0.022\right)$. Patients whose postCABG 24hours TXA-M was in the intermediate tertile had an elevated HR of 2.22 in comparison to patients generating lower level of post-CABG 24 hours TXA-M (95\% CI 1.04 to $4.71, \mathrm{P}=0.038)$, and the higher tertiles of post-CABG 24hours TXA-M bear an even higher HR of $2.81(95 \%$ CI 1.35 to $5.85, \mathrm{P}=0.006)$. Further, we adjusted the model by multiple variables listed in online supplementary table S1. After adjusting, post-CABG 24hours generation of TXA-M still exhibited significant association with MACCE $\left(\mathrm{P}_{\text {trend }}=0.018\right)$. MACCE risk of patients who generated intermediate level of post-CABG 24hours urinary TXA-M was $2.67(95 \%$ CI 1.20 to $5.90, \mathrm{P}=0.016)$ times higher than those who generated low level of post-CABG 24 hours TXA-M, and high tertile of TXA-M generation resulted in a risk 2.86 (95\% CI 1.34 to $6.13, \mathrm{P}=0.007$ ) times more than the low tertile (table 4 , figure 2 ).

In addition, we calculated the C-statistics of the models. Under logistic regression, the C-statistic of clinical factors containing all variables listed in online supplementary table S1 was 0.64 (95\% CI 0.55 to 0.72 ), the C-statistic of post-CABG 24hours TAX-M was 0.62 (95\% CI 0.54 to 0.70). Combining post-CABG 24 hours TAX-M with clinical factors, the C-statistic increased to 0.68 (95\% CI 0.60 to 0.76 ), which indicated the predictive value was improved by adding post-CABG 24 hours TAX-M (online supplementary table S2). 
Table 1 Baseline characteristics of study participants

\begin{tabular}{|c|c|c|c|c|}
\hline & \multirow[b]{2}{*}{ All patients $(n=224)$} & \multicolumn{2}{|l|}{ MACCE } & \multirow[b]{2}{*}{$P$ values } \\
\hline & & No $(n=168)$ & Yes $(n=56)$ & \\
\hline \multicolumn{5}{|l|}{ Age } \\
\hline Mean - years & $61.7( \pm 7.9)$ & $61.7( \pm 7.8)$ & $61.7( \pm 8.2)$ & 0.981 \\
\hline \multicolumn{5}{|l|}{ Distribution - no. (\%) } \\
\hline$\leq 60$ years & $100(44.6)$ & $75(44.6)$ & $25(44.6)$ & 1 \\
\hline$>60$ years & $124(55.4)$ & $93(55.4)$ & $31(55.4)$ & 1 \\
\hline Male sex - no. (\%) & $180(80.4)$ & $136(81.0)$ & $44(78.6)$ & 0.698 \\
\hline Body mass index $\left(\mathrm{kg} / \mathrm{m}^{2}\right)$ & $25.9( \pm 2.9)$ & $25.9( \pm 2.8)$ & $25.9( \pm 3.2)$ & 0.970 \\
\hline Current smoking - no. (\%) & $133(59.4)$ & $103(61.3)$ & $30(53.6)$ & 0.307 \\
\hline \multicolumn{5}{|l|}{ Medical history - no. (\%) } \\
\hline Hypertension & $159(71.0)$ & $121(72.0)$ & 38 (67.9) & 0.552 \\
\hline Diabetes mellitus & $83(37.1)$ & $59(35.1)$ & $24(42.9)$ & 0.299 \\
\hline Chronic obstructive pulmonary disease & $2(0.9)$ & $1(0.6)$ & $1(1.8)$ & 0.412 \\
\hline Peripheral vascular disease & $7(3.1)$ & $5(3.0)$ & $2(3.6)$ & 0.825 \\
\hline Prior MI & $76(33.9)$ & $55(32.7)$ & $21(37.5)$ & 0.515 \\
\hline Chronic kidney disease & $3(1.3)$ & $3(1.8)$ & $0(0.0)$ & 0.314 \\
\hline Ejection fraction (\%) & $60.5( \pm 7.6)$ & $60.5( \pm 7.6)$ & $60.4( \pm 7.9)$ & 0.923 \\
\hline Rosuvastatin use in the STICS trial & $110(49.1 \%)$ & $84(50.0 \%)$ & $26(46.4 \%)$ & 0.643 \\
\hline \multicolumn{5}{|l|}{ Medication use after discharge - no. (\%) } \\
\hline Aspirin & $217(96.9)$ & $163(97.0)$ & $54(96.4)$ & 0.825 \\
\hline$\beta$-blocker & $163(72.8)$ & $124(73.8)$ & $39(69.6)$ & 0.544 \\
\hline Statins & $158(70.5)$ & $120(71.4)$ & $38(67.9)$ & 0.612 \\
\hline ACEI & $52(23.2)$ & $40(23.8)$ & $12(21.4)$ & 0.715 \\
\hline Calcium channel blocker & $52(23.2)$ & $36(21.4)$ & $16(28.6)$ & 0.273 \\
\hline \multicolumn{5}{|l|}{ Scheduled surgery - no. (\%) } \\
\hline On-pump procedure & $101(45.1)$ & $72(42.9)$ & $29(51.8)$ & 0.245 \\
\hline Off-pump procedure & $123(54.9)$ & $96(57.1)$ & 27 (48.2) & 0.245 \\
\hline
\end{tabular}

Values are mean $( \pm S D)$ or $n(\%)$.

ACEI, ACE inhibitors; MACCE, major adverse cardiac and cerebrovascular events; MI, myocardial infarction; STICS, Statin Therapy in Cardiac Surgery.

\section{DISCUSSION}

In this study, we used a nested case-control design to analyse the association between perioperative urinary TXA-M and MACCE in a CABG cohort. According to our results, postCABG 24 hours urinary TXA-M shows significant association of 1year MACCE. Post-CABG 24hours TXA-M of patients with MACCE is significantly higher than that of patients without MACCE. In addition, risk stratification according to the post-CABG 24 hours urinary TXA-M level predicts 1 year
MACCE after CABG. Patient whose post-CABG 24hours urinary TXA-M is in the highest tertile bears almost three times higher risk than those in the lowest tertile. These results indicate that post-CABG 24 hours urinary TXA-M has the potential to be a risk predictor of adverse events after CABG in future clinical practice.

$\mathrm{TXA}_{2}$ originates from AA. The first two steps of AA metabolism are the oxidation catalysed by cyclo-oxygenase-1 or cyclo-oxygenase-2. With these two isoforms

Table 2 Urinary thromboxane metabolite (TXA-M) concentrations pre-CABG, post-CABG 6 hours and post-CABG 24 hours in patients with or without MACCE

\begin{tabular}{|c|c|c|c|c|c|c|c|}
\hline & \multicolumn{3}{|l|}{ MACCE } & \multicolumn{3}{|c|}{ Non-MACCE } & \multirow[b]{2}{*}{$P$ values } \\
\hline & Number & Median & IQR & Number & Median & IQR & \\
\hline Pre-CABG & 56 & 4540 & 2383 6524 & 168 & 5076 & $3398 \sim 7593$ & 0.047 \\
\hline Post-CABG 6 hours & 56 & 24016 & $15541 \sim 35965$ & 168 & 25681 & 17612 35005 & 0.727 \\
\hline Post-CABG 24 hours & 56 & 11101 & 7327 14624 & 168 & 8849 & 5530 12552 & 0.007 \\
\hline
\end{tabular}

CABG, coronary artery bypass graft; MACCE, major adverse cardiac and cerebrovascular events. 
Table 3 Urinary thromboxane metabolite (TXA-M) concentrations of pre-CABG, post-CABG 6 hours and post-CABG 24 hours in control patients and patients who died, patients with stroke, patients with $\mathrm{MI}$ and patients with repeat revascularisation

\begin{tabular}{llllllll}
\hline & Number & Pre-CABG & P values & $\begin{array}{l}\text { Post-CABG } \\
\text { 6hours }\end{array}$ & P values & $\begin{array}{l}\text { Post-CABG } \\
\text { 24hours }\end{array}$ & P values \\
\hline $\begin{array}{l}\text { Patients with } \\
\text { MACCE }\end{array}$ & & & & & & \\
Control patients & 168 & $\begin{array}{l}5076 \\
(3398 \sim 7593)\end{array}$ & - & $\begin{array}{l}25681 \\
(17612 \sim 35005)\end{array}$ & - & $\begin{array}{l}8849 \\
(5530 \sim 12552)\end{array}$ & - \\
Death & 16 & $\begin{array}{l}4879 \\
(1680 \sim 9325)\end{array}$ & 0.596 & $\begin{array}{l}26689 \\
(20423 \sim 26689)\end{array}$ & 0.441 & $\begin{array}{l}11993 \\
(8614 \sim 23384)\end{array}$ & 0.039 \\
Stroke & 26 & $\begin{array}{l}4583 \\
(2713 \sim 5875)\end{array}$ & 0.197 & $\begin{array}{l}26156 \\
(13426 \sim 36372)\end{array}$ & 0.847 & $\begin{array}{l}11138 \\
(8764 \sim 15021)\end{array}$ & 0.016 \\
MI & 2 & $\begin{array}{l}2550 \\
(-)\end{array}$ & 0.075 & $\begin{array}{l}17029 \\
(-)\end{array}$ & 0.225 & $\begin{array}{l}13585 \\
(-)\end{array}$ & 0.444 \\
Revascularisation & 12 & $\begin{array}{l}3384 \\
(2554 \sim 6765)\end{array}$ & 0.931 & $\begin{array}{l}21502 \\
(12965 \sim 32440)\end{array}$ & 0.335 & $\begin{array}{l}8059 \\
(6577 \sim 13642)\end{array}$ & 0.931 \\
\hline
\end{tabular}

Values are median (IQR).

CABG, coronary artery bypass graft; MI, myocardial infarction; MACCE, major adverse cardiac and cerebrovascular events.

of cyclo-oxygenase, AA is synthesised into hydroperoxy endoperoxide PGG2 and its subsequent reduction to the hydroxy endoperoxide PGH2, which would be transformed by TXA synthase into TXA2. ${ }^{14}$ TXA $_{2}$ has a very short half-life and undergoes hydrolysis to the inactive $\mathrm{TXB}_{2}$ without enzyme, then further metabolises to TXA-M (11-dehydro- $\mathrm{TXB}_{2}$ and 11-dehydro-2,3-dinor $\mathrm{TXB}_{2}$ ) and is excreted in the urine. In previous studies, TXA-M has been reported to be associated with increased MI, stroke or cardiovascular death rate in patients with high cardiovascular risk. ${ }^{15}$ Moreover, TXA-M also correlates with the increase of cardiovascular events and mortality in patients with atrial fibrillation. ${ }^{16}$ For patients with $\mathrm{CABG}$, the urinary TXA-M level is associated with SVG patency. ${ }^{4}$ Our study demonstrates that perioperative TXA-M level is associated with adverse events in the first year after CABG for the first time.

In our study, pre-CABG TXA-M has a marginally significant association with 1 year MACCE after CABG; patients who generate high levels of TXA $_{2}$ tend to have low risks of MACCE. As the P value of this association is marginal, further study should be undertaken out to confirm this correlation. Urinary TXA-M increases sharply at 6 hours after CABG, which may be caused by the turnover of newly generated platelets and increased inflammation induced by the surgical procedure. At 24 hours after CABG, urinary TXA-M decreases compared with postCABG 6 hours urinary TXA-M, which is mainly caused by decreased inflammatory response. However, the generation of urinary TXA-M still remains at a relatively high

Table 4 Cox regression analysis of MACCE according to levels of pre-CABG, post-CABG 6 hours and post-CABG 24 hours urinary thromboxane metabolite (TXA-M)

\begin{tabular}{|c|c|c|c|c|c|c|c|}
\hline & \multirow[b]{2}{*}{ Tertile } & \multicolumn{3}{|c|}{ Unadjusted } & \multicolumn{3}{|c|}{ Adjusted* } \\
\hline & & HR & $95 \% \mathrm{Cl}$ & $P$ values & HR & $95 \% \mathrm{Cl}$ & $P$ values \\
\hline \multirow[t]{3}{*}{ Pre-CABG } & 1 & - & - & 0.075 & - & - & 0.066 \\
\hline & 2 & 0.54 & $0.28 \sim 1.01$ & 0.055 & 0.52 & $0.27 \sim 1.03$ & 0.062 \\
\hline & 3 & 0.55 & $0.29 \sim 1.03$ & 0.062 & 0.48 & $0.25 \sim 0.95$ & 0.036 \\
\hline \multirow[t]{3}{*}{ Post-CABG 6 hours } & 1 & - & - & 0.755 & - & - & 0.564 \\
\hline & 2 & 1.21 & $0.64 \sim 2.27$ & 0.556 & 1.33 & $0.68 \sim 2.22$ & 0.404 \\
\hline & 3 & 0.92 & $0.50 \sim 1.88$ & 0.923 & 0.94 & $0.46 \sim 1.93$ & 0.862 \\
\hline \multirow[t]{3}{*}{ Post-CABG 24 hours } & 1 & - & - & 0.022 & - & - & 0.018 \\
\hline & 2 & 2.22 & $1.04 \sim 4.71$ & 0.038 & 2.67 & $1.20 \sim 5.90$ & 0.016 \\
\hline & 3 & 2.81 & $1.35 \sim 5.85$ & 0.006 & 2.86 & $1.34 \sim 6.13$ & 0.007 \\
\hline
\end{tabular}

Adjusted by all the variables listed in online supplementary table S1 (age, sex, body mass index, current smoking, hypertension, diabetes mellitus, chronic obstructive pulmonary disease, peripheral vascular disease, prior myocardial infarction, chronic kidney disease, ejection fraction, on-pump procedure, aspirin, $\beta$-blocker, statins, ACE inhibitors, calcium channel blocker).

CABG, coronary artery bypass graft; MACCE, major adverse cardiac and cerebrovascular events. 

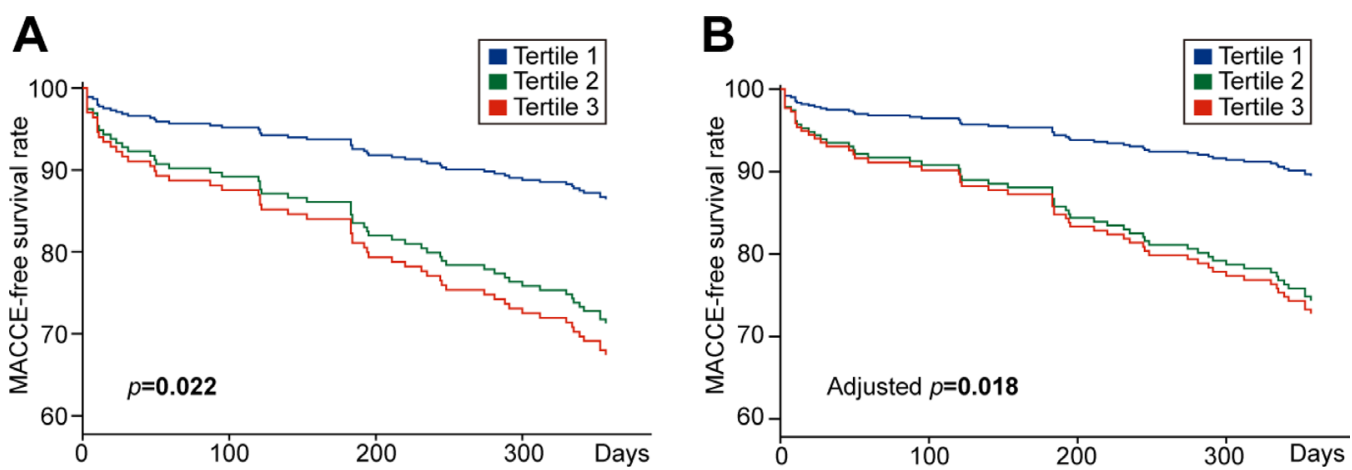

Figure 2 Kaplan-Meier curves of MACCE-free survival rate according to post-CABG 24 hours concentration of urinary thromboxane metabolite (TAX-M). Analysis of MACCE-free survival rate according to concentration tertiles of post-CABG 24 hours urinary TAX-M. (A) Unadjusted curve of MACCE-free survival rate; (B) Curve of MACCE-free survival rate adjusted by variables listed in online supplementary table S1. CABG, coronary artery bypass graft; MACCE, major adverse cardiac and cerebrovascular events.

level 24 hours after CABG compared with the normal status. The main reason for this phenomenon is the cyclo-oxygenase-2-derived thromboxane synthesis. Under normal conditions, an overwhelming majority of $\mathrm{TXA}_{2}$ is generated by platelet cyclo-oxygenase-1. As an isoform of cyclo-oxygenase, cyclo-oxygenase-2 is mainly activated during the inflammatory process and has been reported to play important roles in the process of atherosclerosis and the progression of CAD. In response to the inflammatory stimuli triggered by the CABG procedure, ${ }^{17} 18$ the expression of cyclo-oxygenase-2 is augmented 10-20fold in nucleated cells. ${ }^{14}{ }^{15}$ Besides, platelet turnover enhanced by the CABG procedure also elevates platelet cyclo-oxygenase-2 expression. ${ }^{19} 20$ Aspirin irreversibly inhibits cyclo-oxygenase by acetylating serine 530 of cyclo-oxygenase-1 or serine 516 of cyclo-oxygenase- $2,{ }^{21-23}$ but cyclo-oxygenase-2 is $\sim 170$-fold less sensitive to inhibition by aspirin than cyclo-oxygenase- $1 .{ }^{24}$ So the cyclo-oxygenase-2-derived thromboxane synthesis cannot be effectively inhibited by aspirin, which may contribute to the increased MACCE rate after CABG.

Apart from cardiac surgery, TXA-M has also been described as a potential predictive biomarker of MACCE in patients with high cardiac risk and in patients with acute MI. Eikelboom et $a l^{15}$ first found that urinary concentrations of TXM predicted future risk of MI or cardiovascular death. These findings raised the possibility that elevated urinary TXM levels identify patients who are relatively resistant to aspirin and who may benefit from additional antiplatelet therapies or treatments that more effectively block in vivo thromboxane production or activity found in aspirin-treated patients. Then, Eikelboom et $a l^{25}$ suggested that urinary concentrations of TXM are an externally valid and potentially modifiable determinant of stroke, MI, or cardiovascular death in patients at risk for atherothrombotic events. In patients with acute MI, Szczeklik et $a l^{26}$ found that urine TXM could predict 1 year MACCE in patients with acute MI and provide prognostic information on left ventricular performance.
Although, our results indicate urinary TXA-M has the potential to be a risk predictor of adverse events after CABG, this study has several potential limitations. First, the stability of the high urinary TXA-M excretion phenotype is unknown. In other words, we have not tested TXA-M levels at later time points beyond the first day after CABG, thus, whether the TXA-M level in this study is a temporary change or a stable one should be verified in further studies. Second, as a nested case-control study, we did not test TXA-M levels for all patients in the STICS cohort, which mildly reduces the strength of our findings. So a further prospective study focused on this issue should be undertaken to validate our findings.

In summary, in a nested case-control study, we conclude that the perioperative urinary TXA-M level is associated with 1 year adverse events after CABG, which raises the potential possibility that high levels of perioperative TXA-M identify patients with high risk of post-CABG adverse events.

Contributors $\mathrm{HL}$ and $\mathrm{ZX}$ were involved in data collection, data analysis, writing of manuscript. CS and NB were involved in data verification. WC and QC were involved in the collection of patients' urine and test of TXA-M in patients' urine. ZhoZ, ZheZ and XW were involved in the design of study and student supervision, editing of manuscript.

Funding This study was supported by the Key Project in the National Science and Technology Pillar Programme during the 12th 5-year plan period (2013BAI09B01).

Competing interests None declared.

Patient consent Not required.

Ethics approval Review Board of Fuwai Hospital, Peking Union Medical College (Beijing, China)

Provenance and peer review Not commissioned; externally peer reviewed. Data sharing statement № additional data are available.

Open access This is an open access article distributed in accordance with the Creative Commons Attribution Non Commercial (CC BY-NC 4.0) license, which permits others to distribute, remix, adapt, build upon this work non-commercially, and license their derivative works on different terms, provided the original work is properly cited, appropriate credit is given, any changes made indicated, and the use is non-commercial. See: http:// creativecommons.org/licenses/by-nc/4.0/. 


\section{REFERENCES}

1. Piccolo R, Giustino G, Mehran R, et al. Stable coronary artery disease: revascularisation and invasive strategies. Lancet 2015;386:702-13.

2. Torsney E, Mayr U, Zou Y, et al. Thrombosis and neointima formation in vein grafts are inhibited by locally applied aspirin through endothelial protection. Circ Res 2004;94:1466-73.

3. Halabi AR, Alexander JH, Shaw LK, et al. Relation of early saphenous vein graft failure to outcomes following coronary artery bypass surgery. Am J Cardiol 2005;96:1254-9.

4. Gluckman TJ, McLean RC, Schulman SP, et al. Effects of aspirin responsiveness and platelet reactivity on early vein graft thrombosis after coronary artery bypass graft surgery. J Am Coll Cardiol 2011;57:1069-77.

5. Xiao CY, Hara A, Yuhki K, et al. Roles of prostaglandin I(2) and thromboxane $A(2)$ in cardiac ischemia-reperfusion injury: a study using mice lacking their respective receptors. Circulation 2001;104:2210-5.

6. Kobayashi T, Tahara Y, Matsumoto M, et al. Roles of thromboxane $\mathrm{A}(2)$ and prostacyclin in the development of atherosclerosis in apoEdeficient mice. J Clin Invest 2004;114:784-94.

7. Cheng Y, Austin SC, Rocca B, et al. Role of prostacyclin in the cardiovascular response to thromboxane A2. Science 2002;296:539-41.

8. Smyth EM. Thromboxane and the thromboxane receptor in cardiovascular disease. Clin Lipidol 2010;5:209-19.

9. Arazi HC, Doiny DG, Torcivia RS, et al. Impaired anti-platelet effect of aspirin, inflammation and platelet turnover in cardiac surgery. Interact Cardiovasc Thorac Surg 2010;10:863-7.

10. Zheng Z, Jayaram R, Jiang L, et al. Perioperative Rosuvastatin in Cardiac Surgery. N Engl J Med 2016;374:1744-53.

11. Serruys PW, Morice MC, Kappetein AP, et al. Percutaneous coronary intervention versus coronary-artery bypass grafting for severe coronary artery disease. N Engl J Med 2009;360:961-72.

12. Zhang $\mathrm{H}$, Yuan $\mathrm{X}$, Zhang $\mathrm{H}$, et al. Efficacy of Long-Term $\beta$-Blocker Therapy for Secondary Prevention of Long-Term Outcomes After Coronary Artery Bypass Grafting Surgery. Circulation 2015;131:2194-201.

13. Ames PR, Batuca JR, Muncy IJ, et al. Aspirin insensitive thromboxane generation is associated with oxidative stress in type 2 diabetes mellitus. Thromb Res 2012;130:350-4.
14. Fontana P, Zufferey A, Daali Y, et al. Antiplatelet therapy: targeting the TxA2 pathway. J Cardiovasc Trans/ Res 2014;7:29-38.

15. Eikelboom JW, Hirsh J, Weitz JI, et al. Aspirin-resistant thromboxane biosynthesis and the risk of myocardial infarction, stroke, or cardiovascular death in patients at high risk for cardiovascular events. Circulation 2002;105:1650-5.

16. Pastori D, Pignatelli $P$, Farcomeni A, et al. Urinary 11-dehydrothromboxane B2 is associated with cardiovascular events and mortality in patients with atrial fibrillation. Am Heart $J$ 2015;170:490-7.

17. Rimmelé T, Venkataraman R, Madden NJ, et al. Comparison of inflammatory response during on-pump and off-pump coronary artery bypass surgery. Int J Artif Organs 2010;33:131-8.

18. Bicer M, Senturk T, Yanar M, et al. Effects of off-pump versus onpump coronary artery bypass grafting: apoptosis, inflammation, and oxidative stress. Heart Surg Forum 2014;17:271-6.

19. Rocca B, Secchiero P, Ciabattoni G, et al. Cyclooxygenase-2 expression is induced during human megakaryopoiesis and characterizes newly formed platelets. Proc Natl Acad Sci USA 2002;99:7634-9.

20. Hankey GJ, Eikelboom JW. Aspirin resistance. Lancet 2006;367:606-17.

21. Awtry EH, Loscalzo J. Aspirin. Circulation 2000;101:1206-18.

22. Roth GJ, Majerus PW. The mechanism of the effect of aspirin on human platelets. I. Acetylation of a particulate fraction protein. J Clin Invest 1975;56:624-32.

23. Loll PJ, Picot D, Garavito RM. The structural basis of aspirin activity inferred from the crystal structure of inactivated prostaglandin $\mathrm{H} 2$ synthase. Nat Struct Biol 1995;2:637-43.

24. Vane JR, Bakhle YS, Botting RM. Cyclooxygenases 1 and 2. Annu Rev Pharmacol Toxicol 1998;38:97-120.

25. Eikelboom JW, Hankey GJ, Thom J, et al. Incomplete inhibition of thromboxane biosynthesis by acetylsalicylic acid: determinants and effect on cardiovascular risk. Circulation 2008;118:1705-12.

26. Szczeklik W, Stodółkiewicz E, Rzeszutko M, et al. Urinary 11-Dehydro-Thromboxane B2 as a Predictor of Acute Myocardial Infarction Outcomes: Results of Leukotrienes and Thromboxane In Myocardial Infarction (LTIMI) Study. J Am Heart Assoc 2016;5:e003702. 